\title{
Social weight: designing to minimise the social consequences arising from technology use by the mobile professional
}

Received: 24 February 2003 / Accepted: 2 April 2003/Published online: 16 September 2003

(C) Springer-Verlag London Limited 2003

\begin{abstract}
This paper defines the concept of social weight as a design consideration and presents the e-SUIT, a social weight research platform incorporated covertly within a traditional business suit. The e-SUIT allows its user to strike a balance between a given technology's derived benefit and its social consequence. As the eSUIT is designed for research within a business context, it is built upon commercially available enterprise software. This work is a first step towards subjecting the empirical social interactive phenomena of wearable technology to quantitative design analysis. Proof of concept testing shows access to commercially available enterprise applications with a distinct, user selectable, strata of social weight.
\end{abstract}

Keywords Social weight $\cdot$ Mobile professional Wearable

\section{Introduction}

Current metrics of technology achievement focus on gains in efficacy unmitigated by resultant social consequence. This paper expands upon our work "Minimum social weight user interactions for wearable computers in business suits" presented at the International Symposium on Wearable Computers (ISWC) 2002, defining the concept of social weight as a design consideration and presenting the e-SUIT, a social weight research platform incorporated covertly within a traditional business suit. The social weight for an item of technology can be defined as the attenuation of social interaction that an item causes between its user and others. The e-SUIT incorporates a system that allows its user to strike a balance

A. Toney $(\bowtie) \cdot$ B. Mulley $\cdot$ B. H. Thomas $\cdot$ W. Piekarski School of Computer and Information Science,

Wearable Computer Laboratory,

University of South Australia, Mawson Lakes Boulevard, Mawson Lakes, SA, 5095, Australia

E-mail: joeboy@hhhh.org between a given technology's functionality and its social impact. As a flexible platform for measuring the social weight of its component interfaces, the e-SUIT provides a method to gauge its interfaces' corresponding interpersonal consequences. The ultimate goal of this work is to characterise factors defining social weight with the eventual creation of design constraints, which will lead to devices with a higher degree of social transparency.

Research efforts presented within this paper represent the first attempt to characterise the function of social weight and its major components. These characterisations are constructed under the assumption there are known and yet to be discovered components forming social weight. The business environment was chosen as the setting for initial social weight research because its interactions are frequently of a high personal and economic importance while taking place on a well-defined conservative social foundation. The highly structured social contexts provided by the business world minimise the impact of unknown independent variables that effect the formation of social weight. As a result, the business environment presents an ideal arena for social weight research.

To make the e-SUIT useful within the context of the business community, it needs to be physically discrete while at the same time interfacing seamlessly with existing commercial grade business information systems. By integrating the technologies required directly into the user's clothing, the e-SUIT achieves a significant level of physical discretion. Figure 1 shows a user covertly entering a change to his electronic calendar appointment via the user interface device sewn into the right hem of the e-SUIT jacket. The required integration with existing business information systems is provided by interacting within commercial personal information managers (PIMs), such as Microsoft Outlook. The user interaction technology presented in this paper allows users to interact with their own Windows Outlook information system in a minimum social weight fashion. These technologies are not limited to this application, but demonstrate a compatibility with existing commercial software systems. 


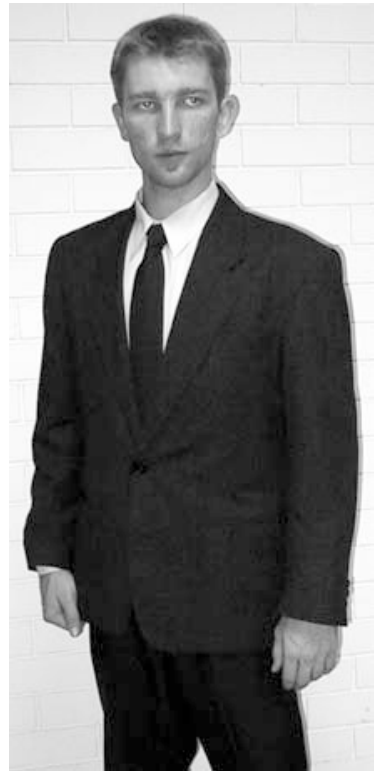

Fig. 1 The e-SUIT

In a business setting, people often interact with other people in many different locations over the course of a business day. The initial research into social weight presented within this paper focuses on situations where the user is away from the office or workspace. It is in these situations that mobile computing and communication devices are most useful, and the user generally finds the least accessible infrastructure. The thin clients which typically dominate in these sorts of areas (e.g., pagers, PIMs, PDAs, etc.) represent devices that are designed to be able to be used concurrently with social interaction. The requirements of this social concurrency make the devices particularly susceptible to social consequence. As such, the overall benefit provided by any of these devices is heavily mitigated by its respective social weight. The e-SUIT mirrors the functionality of some these devices to provide a research platform for mobile use where social weight is most significant.

The remainder of this section describes our assertions for a business suit, presents a description of notificationbased systems and their current known interface problems and introduces social weight with an explanation of it role and significance. We then describe the new user interface controls for the e-SUIT, and the e-SUIT's implementation details. A discussion then focuses on the successes of the e-SUIT and outlines the areas requiring further work. Required future research is then presented, and the paper finishes with concluding remarks and acknowledgements.

\subsection{Business suits and other uniforms}

We assert that new wearable technologies to be incorporated into business wear will have to be designed to fit the uniform of the business suit. Men's business suits, which include a jacket, shirt, tie, trousers and sometimes a vest, have been worn for almost two centuries, and they have been of a consistent design [5].

In the past, wearable computers have been incorporated into a number of different workplace attires, such as battlefield attire [10], maintenance attire [17], and field observation attire [13]. As previously mentioned, we are investigating the incorporation of a wearable computer within a business suit. The wearable computers for battlefield, maintenance, and field observation attires were visibly integrated into the apparel, and the functionality of the computing systems was promoted at the expense of fashion. Sheridan et al. [16] reported that the public perception of cyborgs (the user's of unconcealed wearable computers) is tentative at best and, more often than not, overtly negative.

In the case of a business suit, fashion plays a much more important role. To this end, we propose that a wearable computer, which is part of the suit, should be invisible to other people unless the user chooses to use the more overt aspects of the computer. This removes the need for us to design a fashionable looking wearable computer (we leave this to the fashion designers). Also, a piece of technology that is not noticeable to other people has an inherent low social weight. As a case in point, mobile phones have gotten smaller over the years. While the phones have been increasing in functionality, users have been taking phones off their belts and placing them into their pockets. In the case of phones, it would seem people are not changing the way they look to fit their technology.

\subsection{Interruptions, disruptions and notifications}

There is a range of applications that require some form of notification; some examples are alarms, reminders, e-mail, instant messaging and calendars. Reminders are a form of notification that have the following two characteristic features: a signal to indicate something is to be remembered and a description to explain what needs to be remembered [4]. Current reminder systems, acting as a form of externalised memory, do not present appropriate signals at all times. More specifically, these tools are not sufficient because they are not proactive and do not make use of rich contextual information to trigger reminders at appropriate times in appropriate locations. Herstad et al. [8] claim that in order to build useful, functional and powerful tools for supporting human-human interaction, we must take context into account. CybreMinder [4] is a desktop system that determines what is the most appropriate delivery mechanism for each reminder recipient. Dey and Abowd list a set of the features an ideal reminder tool should support: the use of rich context for specifying reminders, the ability for users and third parties to submit reminders, the ability to create reminders using a variety of input devices, the ability to receive reminders using a variety of devices, the use of reminders that include both 
a signal and a full description and allowing users to view a list of all active reminders.

Notifications may interrupt and disrupt a user's current task and social interactions. Recent research has shown notifications from instant messaging to have a generally disruptive effect during fast, stimulus-driven search tasks [3]. As an example, a user is in a meeting with a group of people and his mobile phone rings. The noise of the phone ringing produces a large social weight for the phone; the group will stop talking until the user interacts in some fashion with his phone. Vibrating motors have been introduced to provide a more covert means of informing the user of a phone call or message. The e-SUIT incorporates an extension of this idea to include a larger range of input and output devices to provide a range of interactions, from more covert to more overt, for a user with their computing environment.

Our goal is to provide users with access to a specialised range of functionality while maintaining the covert nature of the devices. If the user chooses to apply more functionality than the covert devices are capable of, the user then relies on his more standard computing devices, such as PDAs and notebook/desktop computers. A significant feature of this design is that the user has control of whether the more overt devices are brought into play. We place the responsibility to perform tasks on the appropriate appliances with an appropriate social weight upon the user.

Herstad et al. [8] field studied three different communication applications in highly mobile and communication intensive activities. Based on their studies they found the following two problems:

1. There is a need for user control of selection of the communication media during mobile communication

2. There is a need for user control of interaction modality when interacting with the communication application.

O'Conaill and Frohlich [12] found that a level of filtering of interruptions would be desirable to determine if the current interruption warranted a disruption of the prior activity. This filtering can be performed with good effect in conjunction with a human personal assistant, e.g. a receptionist or secretary.

\subsection{Social weight}

The social weight $(S W)$ for an item of technology can be defined as the attenuation the item causes in social interaction between its user and others. For the purposes of this paper four components of social weight have been identified: cognitive load $(C L)$, physical presence $(P P)$, technology apprehension $(T A)$, and social convention $(S C)$. Social weight is being treated as an unknown function of these four variables; $S W=f(C L, P P$, $T A, S C)$. The e-SUIT was created to provide a research platform whose social weight was constrained to discreet user selectable levels. Through a controlled selection of the degree of social weight the aspects of social consequence can be selectively emphasised, characterising their effect on the formation of social weight.

\subsubsection{Social context}

The social context of our initial research was restricted to dyadic interactions between the user and a single social partner. Our work has the user interacting with technology within his personal information space. Figure 2 illustrates this paper's operational social context.

\subsubsection{Social convention}

Social conventions (SCs) governing acceptable usage evolve out of society's prolonged contact with technology. While mature SCs have significant social consequence, SCs are largely absent for original new technology. As such, SC presents an independent variable so unconstrained as to present a severe challenge to the characterisation of social weight and its components' interactions.

The e-SUIT's design was predicated on the belief that technology young enough not to have evolved SCs of its own may inherit the conventions of existing familiar technology. Design criteria for the e-SUIT was constrained by the need to minimise the variability of relevant SCs. As a result, one of the design constraints was that where the e-SUIT's incorporated technology was visible to social participants it needed to present an interaction profile similar to existing and familiar devices.

To be successful, any usage of the e-SUIT should present itself as the user merely engaging technology familiar to the observer. For example, when the e-SUIT's watch is used to display and navigate menu information it must appear to onlookers that the e-SUIT's user is merely checking the time or responding to an alarm. Similarly, when the vibrotactile display formed by the pager motors presents information, or

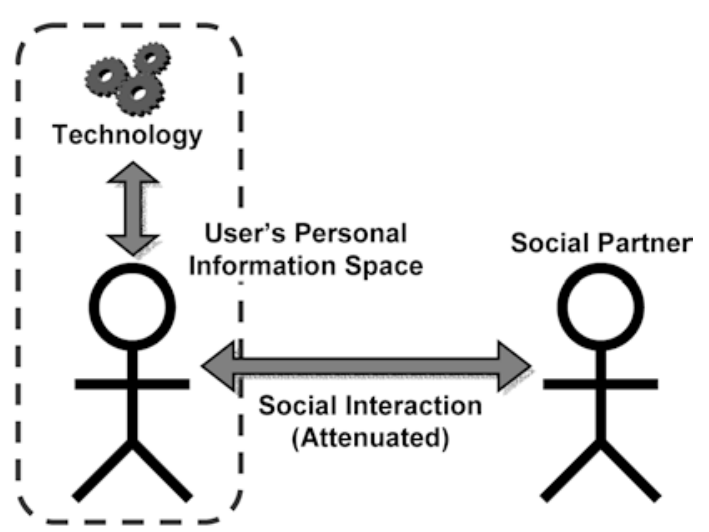

Fig. 2 Social context 
when the integrated keyboard is used, it should not appear that the user is interacting with any technology. At all times, the user of the e-SUIT must either appear to be using conventional technology or not to be using technology at all.

Successfully masquerading as an array of conventional devices means that the e-SUIT will be evaluated under mature SCs. In other words, observers evaluate the usage of the e-SUIT's technology based on whatever devices they believed were being used. While the masquerade impacts the other components of social weight, it allows for the SC to be factored out of the initial data allowing for calculation of a function approximating social weight in three variables: $T A, P P$, and $C L$.

As stated earlier for the purposes of the work presented within this paper $S W=f(C L, P P, T A, S C)$. For devices evaluated under mature SCs a set of discrete convention weightings, $W s c$, can be derived for each interaction modes. So, for each of our scenarios using the e-SUIT a set of weightings $W s c=\left\{W s c_{1}, W s c_{2}, \ldots\right.$, $\left.W s c_{\mathrm{n}}\right\}$ can be derived where $S W$ is linearly approximated by the function $h(W s c, g(C L, P P, T A))$ and where $S W$ is directly proportional to $g(C L, P P, T A)$. The work presented in this paper sought to characterise the function $g(C L, P P, T A)$ as a function providing an indirect measure of social weight. Once obtained, this indicator of social weight can be used to evaluate the e-SUIT's various user interface options. Subsequent work will be required to broaden the indicators of SW from $g(C L$, $P P, T A)$ to $\mathrm{h}(W s c, g(C L, P P, T A))$.

\subsubsection{Approximating $S W$ : calculating $g(T A, P P, C L)$}

The function $g(C L, P P, T A)$ provides an indirect measure of social weight with three independent variables. The e-SUIT was designed to provide its user choices of distinct strata of social weight within which to move. Through selective emphasis of the components of social weight, and measurement of the resultant social consequence, a best estimate of $g(C L, P P, T A)$ can be obtained for the interfaces contained within the e-SUIT. Cognitive load is gauged using the model human processor as described in [2]. We are using loss of eye contact measured in time as an indirect measure of cognitive load. The physical presence of the device is gauged by the device's relative size and form factor. We are not measuring technology apprehension under the assumption that when the technology is invisible to social partners the resultant technology apprehension will be negligible.

\subsubsection{A user-controlled social profile}

The e-SUIT is designed so that the use of its encompassed technology will have a variable, yet known, list of discrete social weights. The e-SUIT gives its user control over the degree of externalisation of device interaction, providing a mechanism for a user to control his social profile. If a person chooses to get progressively more involved with an item of technology, the social weight has distinct strata within which they can move. The natural end point of this progression, as increases in social weight are traded for expanded functionality, is the use of a PDA or laptop. Given the heightened levels of $S W$ accompanying these devices the user may potentially even terminate the social interaction in favour of interacting solely with the technology.

User control over the degree of externalisation of notification signals is a significant means for allowing a user a graceful progression toward devices with higher social weight. The user is able to use standard social protocols to determine the optimal point to switch to the next level of device. An example of this social protocol is that a person must excuse himself from a group, or merely say, "Excuse me" to a single individual, before commencing to interact with their technology. Looking again at the scenario where a mobile phone vibrates during a group meeting, the user is covertly alerted to a call or message. The predicament is that the user does not have sufficient information to determine if this transaction is worth the social weight of a pause or significant enough to warrant a total break in the social interaction.

\section{Using the e-SUIT}

As a social weight research platform, the e-SUIT was built, wherever possible, upon existing commercially available technology. The same principles were applied to the design of applications running on the e-SUIT. Our initial research application is a calendar program to facilitate communication between a user and his human personal assistant [15]. The application is running on a Compaq H3660 series iPAQ running Microsoft's Pocket Outlook software. The iPAQ operates on the Microsoft Pocket PC 2000, a Windows CE 3.0 variant.

The interface options provided by the e-SUIT promote several implementation strategies for minimising social weight. Our initial work is concerned with two strategies. The first strategy is to provide the user with distinct choices as to the level of social weight he wishes to bear. The second strategy is to programmatically choose the current interface providing computer-mediated escalation of social weight resulting from technology use. Examples of both strategies are provided in our discussion of scenarios using the e-SUIT. To see the e-SUIT configuration and a view of the user wearing the e-SUIT, see Figs. 3 and 4.

\subsection{Scenarios using the e-SUIT}

The e-SUIT provides an awareness continuum along a spectrum from one extreme of peripheral awareness information (PAI) to the other of focal awareness information (FAI) [6]. PAI conveys information 


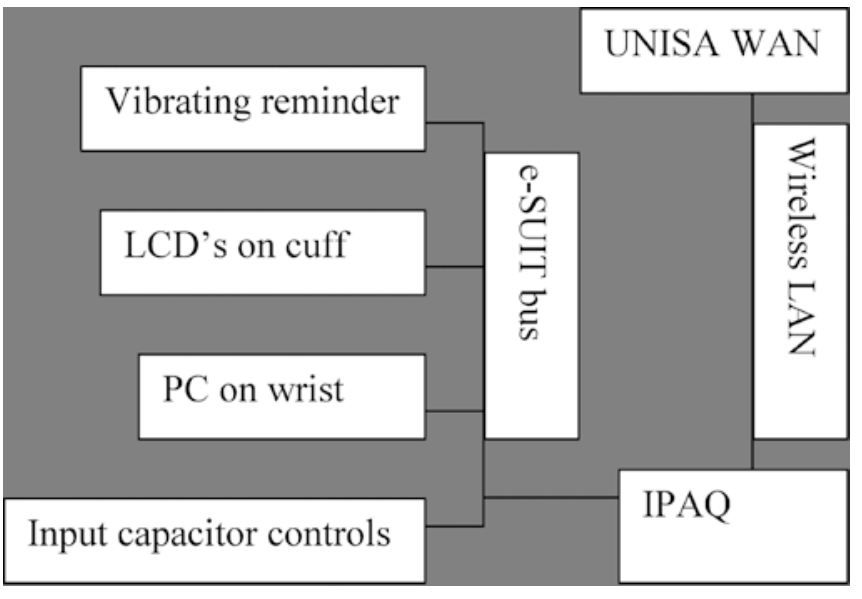

Fig. 3 The e-SUIT configuration

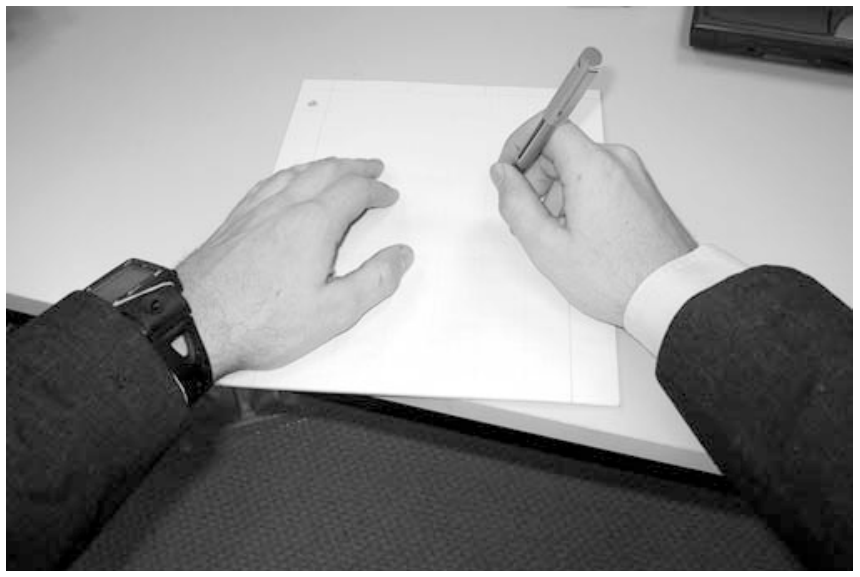

Fig. 4 A view of the user's body while sitting at a table

without requiring the user to take his attention away from other people in a group setting. The concept is to reduce the cognitive effort of the user. Visual, tactile and sound cues can all provide information via the user's peripheral focus of attention. Flashing light emitting diodes (LEDs), vibrating motors in pagers and cellular phones and simple notification chimes or alarms are all examples of PAI. Typically, PAI information is used to draw our attention to potentially more immediate information demands as PAI cues lead to FAI information. FAI is presented more aggressively in a manner commanding a much higher degree of user acknowledgement. Reading an appointment from a PDA or notebook computer are both forms of FAI. As interface options progress from PAI to FAI we see a corresponding increase in SW.

A key assumption underlying all of these usage scenarios is the trust the user has for the integrity of presented information. This is particularly important for highly subjective ratings such as priorities, which are frequently required by information presented to the user. In our application context, the human personal assistant is one of the few people who are able to prioritise appointments for the user. Few people would have the authority to make such an appointment, and as such the user trusts the priority given to appointment.

\subsubsection{Mediating the social impact of mobility}

It is important to recognise that regional or even divisional jobsite groups in an enterprise may cross all factors that determine social weight, e.g., wearing the e-Suit onto the factory floor or into the field for a jobsite. Seamless transition between such radically different environments requires making dynamic changes to the eSuit's user interaction profile. These changes by their very nature require in-depth knowledge of social and environmental context and present an ideal application space for intelligent software agents. Seamless environmental tracking and intelligent agent technology are both research problems within their own right and the authors have chosen to omit them as they are beyond the scope of this paper. While broad in scope all the discussions presented within this paper assume the context provided by the modern office. No agent technologies were developed for the work in this paper. We chose instead to have the user control their interaction profile with the e-Suit.

\subsubsection{The user choice of the strata of the interface social weight}

The user is in a meeting; a vibration alarm in the shoulder of the user's jacket goes off indicating an appointment or incoming message to the user. The user then surreptitiously views a set of LEDs in the cuff of his suit jacket to determine the priority of the information. (The priority to colour mapping is currently controllable by a configuration script; by default red is high, yellow is medium and green is low) Determining it is a high priority piece of information, he casually read a short message off his watch informing him of an important appointment at 2:00 pm that afternoon. The user decides to accept the appointment, but needs to move the appointment two hours forward from the original starting time. He controls the application by manipulating the capacitive controls on the inside of the hem of his suit jacket. The user holds down a button while tapping another to indicate the e-SUIT the appointment should be moved into the future. The outer buttons of the keyboard are pointed to enable the user to easily identify buttons. A unique pulse patterns on the pager motor indicate the currently selected time interval to the user. For this example, the user could tap the button until the pattern indicated two hours into the future. This change in starting time is signalled to the user via a coded pulsing signal from the vibration alarm device.

The user may decide to trade functionality for $S W$ by escalating the interaction to reading his PDA to give a more complete description of the appointment. He then 
may move on to using more intrusive technology, such as a notebook computer or desktop workstation.

\subsubsection{Mediating social impact through priority escalation}

Using the same e-SUIT interfaces as our earlier example a programmatic progression of social weight is also available. Suppose appointments or incoming messages contain both expiration and priority information. Expiration data is present for time-critical information e.g., a message asking the user to call before a certain time or an electronic calendar appointment. Priority information is assigned by trusted individuals e.g., spouses, secretaries and selected co-workers.

The user is standing and having an important conversation with a co-worker; a vibration alarm in the shoulder of the user's jacket goes off indicating an appointment or incoming message. In this case the vibration alarm covertly indicates temporal proximity with the expiration of the message. By the intermittent gentle vibrating pattern the user can tell that the appointment or message will expire within 15 minutes. Knowledge of the time until expiration allows the user to wait for an appropriate point in the conversation to surreptitiously read the cuff of the e-SUIT to determine the priority of the current information.

As the expiration time for the message draws near, the vibration pattern of the tactile alert will become more aggressive and more frequent. While still socially covert from the perspective of social partners, this escalation creates an interface with varying peripheral presence. Such an interface allows the user to have control over both the timing and the degree of social disruption caused by his technology use while still gaining the benefit from device usage. The user can keep interacting with his co-worker while receiving subtle reminders of the upcoming expiration.

Programmatically, controlled escalation of interface provides a clear benefit for mobile applications and has started to be adopted by existing devices such as PDAs that provide progressive alerts as scheduled appointments approach.

Once the user has glanced at the cuff display and determined the message priority, he can wait until a socially appropriate time to casually read a short message off his watch informing him of the subject of the message. For example, the message could read, "Is the development team still meeting at 3:00?" providing the user the choice of "Yes/No/Call". By manipulating the controls on the inside of the hem of his suit jacket the user is able to select the appropriate response, answering the message without overly attenuating or terminating the existing social interaction with his coworker. Alternatively, the user could decide to increase the $S W$ of the interaction by reading his PDA to review his schedule, prior to making his decision. The user may even choose to terminate the existing co-worker conversation to move on to using more socially intrusive technology, such as a notebook computer or desktop workstation. While aspects of interface social weight are programmatically controlled, the user still has the power to control the degree to which he wants his social interactions to be adversely affected by social weight.

\subsection{The social weight of the displays}

Table 1 quantifies the approximate $S W$ ranges for the six different display devices for our system. We measure "model human processor" (MHP) and "loss of eye contact" (LOEC) with time measurements. The following time ranges in seconds quantify the groupings as follows: none $<<1 ; 1 \leq$ small $<2 ; 2 \leq$ medium $<5$; $5 \leq$ large $<15 ; 15 \leq$ very large. As previously mentioned, these values are estimations on our part to provide a means of grouping the $S W$ values of different devices. We based these assumptions on a reading rate of 4.35 words per second (five letters per word). We are assuming a base reaction time to be 0.38 seconds; this is the time to perform the task of seeing a visual stimulus, to access an information chunk from long-term memory to make a decision about the stimulus and to respond by pushing a physical YES or NO button. These values can be found in Card et al. [2].

Our values in Table 1 are based on reacting to a notification signal from a calendar appointment. The pager motor has a small ( 0.5 seconds) value for the notification of a new message, and a medium $(4.0 \mathrm{sec}-$ onds) value for decoding the longest of the six different signals. For cellular phones we assume all calls to be received while the user is in a business context. When receiving a text message phones will bear similar loads to either a pager motor or sound, depending on the alert style which it is using. When used for speech communication, the cellular phone initiates concurrent social contact for the user and thus has a very large MHP load. Given our assumption of operation within a business context we further assume that incoming calls important enough to warrant interruption of the current interaction, and not participatory to that interaction, will be dealt with to completion before the current interaction is resumed. For the calculation of LOEC this means that the user will likely visually be socially engaging the initial participant and thus the phone will have a correspondingly very large LOEC. The physical size of the

Table 1 The docial weight factors for the display devices

\begin{tabular}{llll}
\hline & MHP & $\begin{array}{l}\text { Loss of eye } \\
\text { contact (LOEC) }\end{array}$ & $\begin{array}{l}\text { Physical size of the } \\
\text { device between } \\
\text { users (PSODBU) }\end{array}$ \\
\hline Pager motor & Small/medium & None & None \\
Sound & Small & None & Very large \\
LEDs & Small & Small & Non-visible \\
Wrist PC & Medium & Medium & Small \\
Cell phone & Very large & Very large & Small \\
PDA & Large & Large & Medium \\
Laptop & Very large & Very large & Very large \\
\hline
\end{tabular}


device between users (PSODBU) is based on what we believe to be inconspicuously sized devices. We have one exception to the use of PSODBU. The PSODBU for sound is defined as the impact on people other than the user in a group situation. We place a very large value for PSODBU in the case of sound due to its large disruptive nature in the social situation.

\subsection{The social weight of input devices}

The social weight of input devices can be approximated in terms of human processor time, that is to say, cognitive load. Our premise is that the user interface components that are closer to the PAI side of the awareness continuum, are the preferred controls, due to their lower $S W$. Table 2 quantifies the social weight factors for the four different input devices.

\subsection{Output display devices}

When users are sitting at a meeting table or standing in a public area, the visible features of their own bodies are their forearms, wrists and hands. We have located two visual display devices on these portions of the user's body to reduce head movement when viewing the displays. Coloured LEDs are sewn into the dominanthanded cuff of the e-SUIT jacket. These are placed so as to be visible to the user when viewed straight on. The second visual display device is an LCD programmable watch to display simple text messages. A pager motor is inserted into the shoulder of the e-SUIT to provide a tactile display device. The iPAQ PDA provides the sound display, and the user has the option of removing the iPAQ PDA from their pocket to use a higher resolution display.

\subsubsection{Embedded coloured LEDs}

We have investigated two coding strategies for the cuff LED display. The first is to indicate how much time has elapsed since the user was notified of a message requiring attention. The green LED is lit first, followed by the yellow LED if there has been no response within two minutes; finally, the red LED is lit if there was no response after five minutes. These time values were chosen

Table 2 The social weight factors for the input devices

\begin{tabular}{llll}
\hline MHP & $\begin{array}{l}\text { Loss of eye } \\
\text { contact (LOEC) }\end{array}$ & $\begin{array}{l}\text { Physical size of the } \\
\text { device between } \\
\text { users (PSODBU) }\end{array}$ \\
\hline $\begin{array}{lll}\text { Suit button } \\
\text { Wrist PC }\end{array}$ & $\begin{array}{l}\text { Small/medium } \\
\text { Medium }\end{array}$ & $\begin{array}{l}\text { None } \\
\text { Medium }\end{array}$ & $\begin{array}{l}\text { Non-visible } \\
\text { Small }\end{array}$ \\
PDA & Very large & Very large & Small \\
Laptop & Verge & Large & Medium \\
\end{tabular}

arbitrarily, and further investigation is required to determine optimal values. A second strategy implements the following colour-coding of the priorities for the notifications: red is high, yellow is medium and green is low. These are just two possibilities for colour-coding of the LEDs (Fig. 5 shows the LEDs on a suit cuff).

\subsubsection{The LCD programmable watch}

We are using the onHand watch computer by Matsucom [9] to supply the display device incorporated into a watch. The onHand's display is a 102 by 64 dot-matrix LCD with an EL backlight, supporting 5 lines of 16 characters each. The notification application we have developed for the watch provides its normal timekeeping function, and two lines of 16 characters are overlaid on the top portion of the display (see Fig. 6. The IBM watch computer [11] is an excellent example of the direction this form of interaction technology is heading;

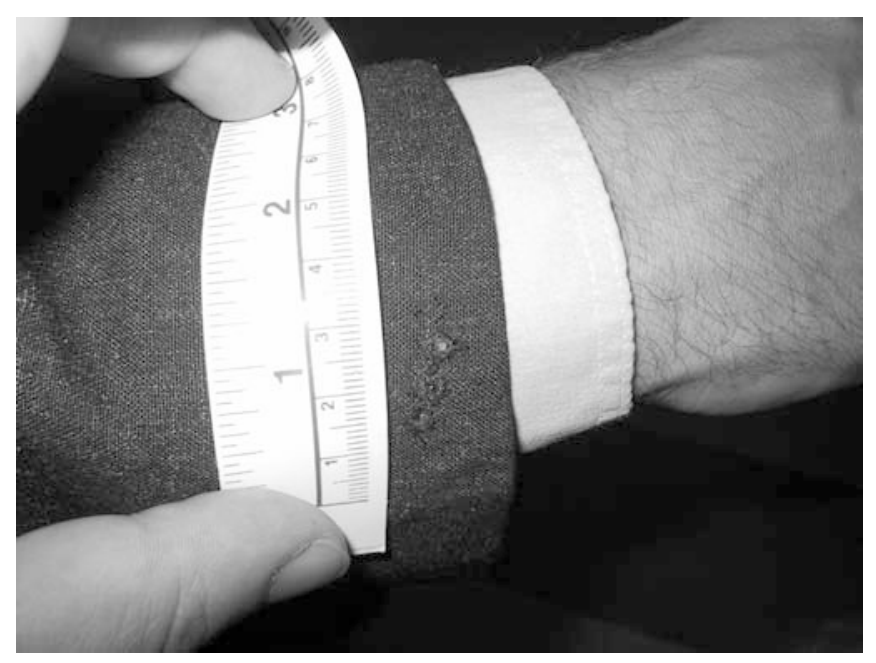

Fig. 5 LEDs on the e-SUIT cuff

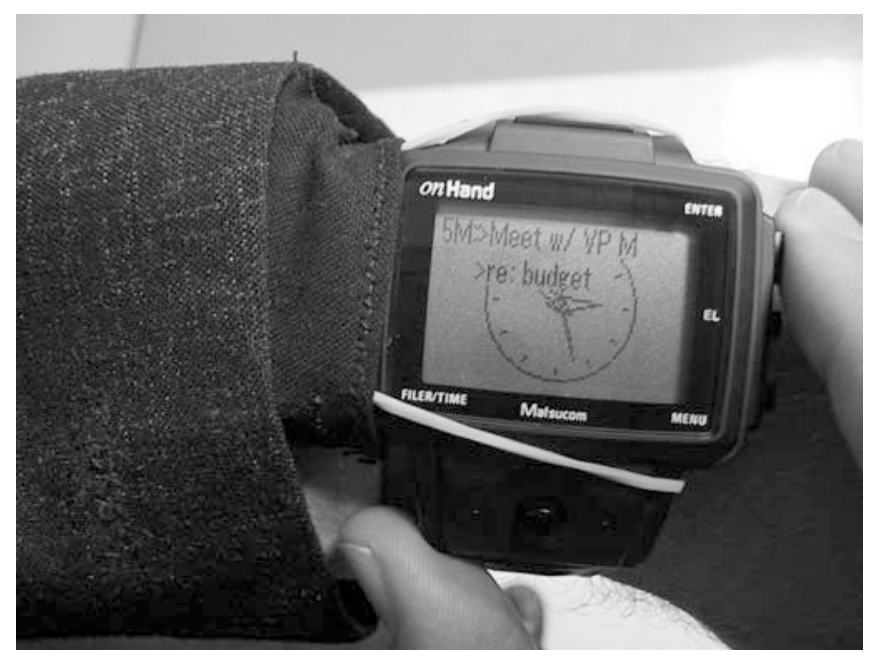

Fig. 6 The watch display 
the embedding of new technology into existing fashionable artefacts currently worn by people.

\subsubsection{The tactile display}

A pager motor is sewn into the shoulder portion of the suit jacket, to maintain pressure between the vibrating motor and the user's body. This display provides a simple one-bit serial display for the user. We have coded 7 simple signals. One signal is a continuous vibration as a notification of a new message. The next 6 coded signals are repeating patterns to indicate the user's specified change to the length of time to move an appointment forward or the length of snooze. Table 3 specifies the different tactile patterns coded to the different time values; the time values are in seconds, and symbol $\uparrow$ indicates the motor is on while $\downarrow$ indicates the motor is off. These patterns, while un-optimised, are capable of covertly displaying information more complex than a simple "a message has arrived." Gemperle et al. proposed a similar idea, with a tactile display incorporated into an earpiece [7].

\subsection{Input devices}

Other researchers, such as those who built the Musical Jacket [14], have investigated the placement of input control devices on and into clothing, but to our knowledge this is the first attempt to place controls into a traditional business suit. Our system provides four different sets of controls for the calendar application. These are also ranked from the highest PAI to the highest FAI as follows:

1. Capacitor buttons and sliders on the clothing; various locations are presented in this paper

2. The buttons in the PC watch are a form of input, but we envision limited use of these in the final system

3 . Once the user has decided to break close social contact with other people, the PDA interface may be used

4. The user may decide to use the devices with the largest $S W$ by interacting with their notebook/desktop interface.

Located inside the suit jacket, sitting opposite the hip, just below the dominant hand, (see Figs. 1 and 7) the e-Suit buttons are located near a relaxed resting place for the hands. We believe this is a good choice of

Table 3 Tactile display patterns

\begin{tabular}{ll}
\hline $5 \mathrm{~min}$ & $0.5 \mathrm{~s} \uparrow, 1 \mathrm{~s} \downarrow$ \\
\hline $15 \mathrm{~min}$ & $0.5 \mathrm{~s} \uparrow, 0.5 \mathrm{~s} \downarrow, 0.5 \mathrm{~s} \uparrow, 1 \mathrm{~s} \downarrow$ \\
$30 \mathrm{~min}$ & $0.5 \mathrm{~s} \uparrow, 0.5 \mathrm{~s} \downarrow, 0.5 \mathrm{~s} \uparrow, 0.5 \mathrm{~s} \downarrow, 0.5 \mathrm{~s} \uparrow, 1 \mathrm{~s} \downarrow$ \\
$1 \mathrm{~h}$ & $1 \mathrm{~s} \uparrow, 1.5 \mathrm{~s} \downarrow$ \\
$2 \mathrm{~h}$ & $1 \mathrm{~s} \uparrow, 0.5 \mathrm{~s} \downarrow, 1 \mathrm{~s} \uparrow, 1.5 \mathrm{~s} \downarrow$ \\
Next day & $1 \mathrm{~s} \uparrow, 0.5 \mathrm{~s} \downarrow, 1 \mathrm{~s} \uparrow, 0.5 \mathrm{~s} \downarrow, 1 \mathrm{~s} \uparrow, 1.5 \mathrm{~s} \downarrow$ \\
\hline
\end{tabular}

position for when the user is standing [18]. We propose a second position of inside the cuff on the non-dominant hand side (the same general area as the watch) for when the user is sitting. The impression we wish the user to give is that they are straightening their cuff or adjusting their jacket, while in reality they are controlling their computer. This placement lets the business user interact with the suit with a reasonably low degree of extra motion and thus should cause correspondingly low levels of additional social weight.

Areas for the placement of the buttons we dismissed are as follows:

1. Inside suit pockets: they are hard to reach and uncomfortable to use

2. Inside pants pockets: they are also hard to use and socially unacceptable

3. On the thigh of the pants leg: we are unable to conceive of socially discreet controls at this location.

The optimal positioning is a key research question that will require further investigation.

\section{The implementation}

The driving force behind all the technology implementations on the e-SUIT were constrained by four factors: the availability of technology, the mass production cost, the required device care and maintenance and the overall social profile in the user's daily life when not using the system. We limited ourselves to the technologies and materials that were available to bring products out on to the market today. Next, we were constrained as to the cost of the technology. Any system that needs to be embedded in garments also needs to add a negligible amount to the cost of the garment. To keep the cost down we designed the suit using only common components and targeting a minimal component count. We estimate that if mass-produced in quantity of $10,000+$, the suit's electronics would have a commercially acceptable price or less then $3 \%$ the cost of the typical target suit. The third constraint was that the embedded portion of the technology be maintainable with the same care that an un-instrumented garment would require. Among other things this would mean that our designs would need to be washable.

\subsection{Pager motors}

Due to their use in pagers and cellular phones, a broad range of small and cheap options in vibration generating motors are commercially available. In order to provide vibrotactile output for the e-Suit a small "pager motor" was sewn into its shoulder. The motor was cylindrical, $6 \mathrm{~mm}$ in diameter and $20 \mathrm{~mm}$ in length including the cam weight. The motor operated at $3 \mathrm{~V}$ drawing $120 \mathrm{~mA}$. The motors vibrate by spinning a cam-shaped counter weight. To operate, the motor needed to be 


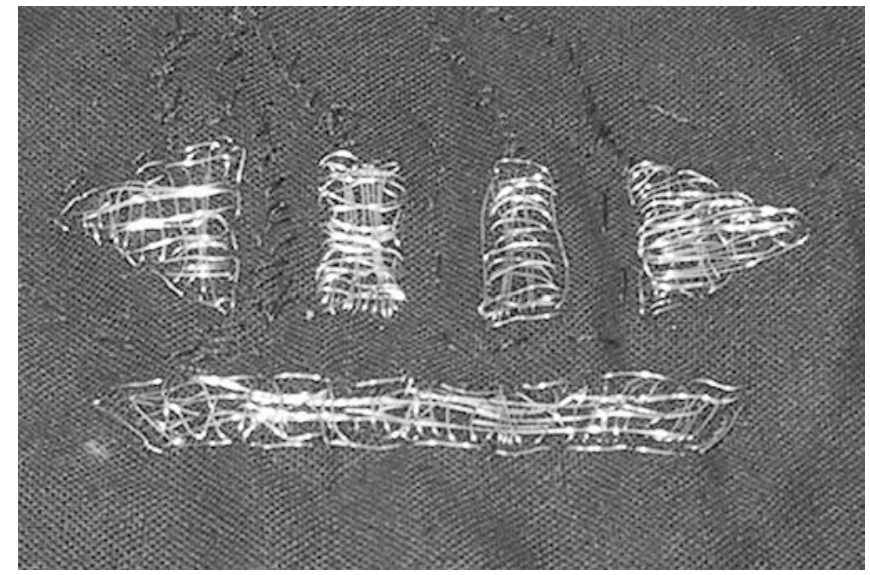

Fig. 7 The e-SUIT's buttons

encased in a cylindrical tube to provide room for the cam to rotate and not be seized by contact with the clothing. This added an additional $2 \mathrm{~mm}$ to the overall diameter. While the shoulder shows great promise as an area for a garment integrated tactile display our initial pager motor implementation was, ultimately, a very low bandwidth communications channel, occasionally uncomfortable to the user due to its shape and rigidity.

\subsection{Capacitive sensors}

The e-Suit buttons are capacitive touch sensors. Fine metal thread suitable for use in tailoring was embroidered into the garment to form the buttons (see Fig. 7); in the future the embroidered patterns could easily be replaced with conductive fabric. When touched, the capacitive value of the embroidered pad increases, potentially by several orders of magnitude. This change in capacitance can be used for the pads to measure proximity, contact, touch pressure, gross finger motion and gesture $[1,14,15]$. The e-Suit currently uses the embroidered pads to implement control buttons and slider functionality. A combination of software and hardware filtering is then performed to filter out spurious environmental triggers.

\subsection{The distribution of power and data}

In order to distribute power and data throughout the jacket we over-sewed, or "bedded", fine wires in the fabric which we could then either sew into the existing garment or use in the creation of new patches with which to amend the garment. The process of bedding the wires is illustrated in Fig. 8. In Fig. 9 and Fig. 10 we show the type of cabling we were using to run through the sleeves of the jacket. The bedding process was to lay the wires down on cloth as desired and then feed these through the sewing machine.

A typical $9 \mathrm{~V}$ battery was used to supply power for all the custom devices in the e-Suit while both the IPAQ

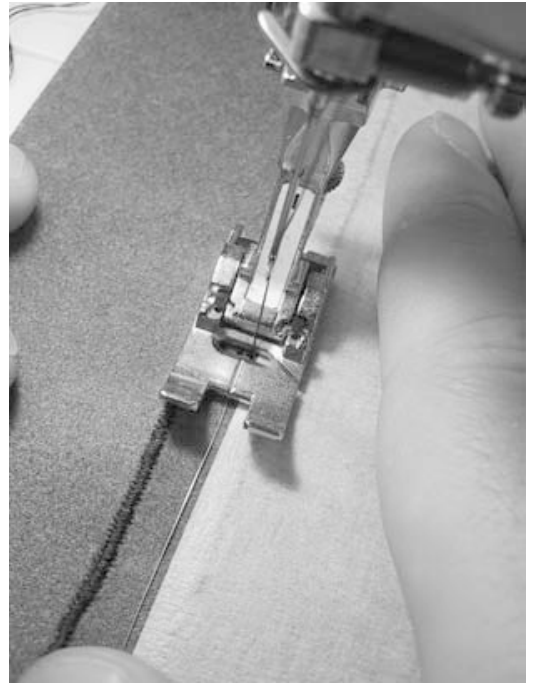

Fig. 8 Run making

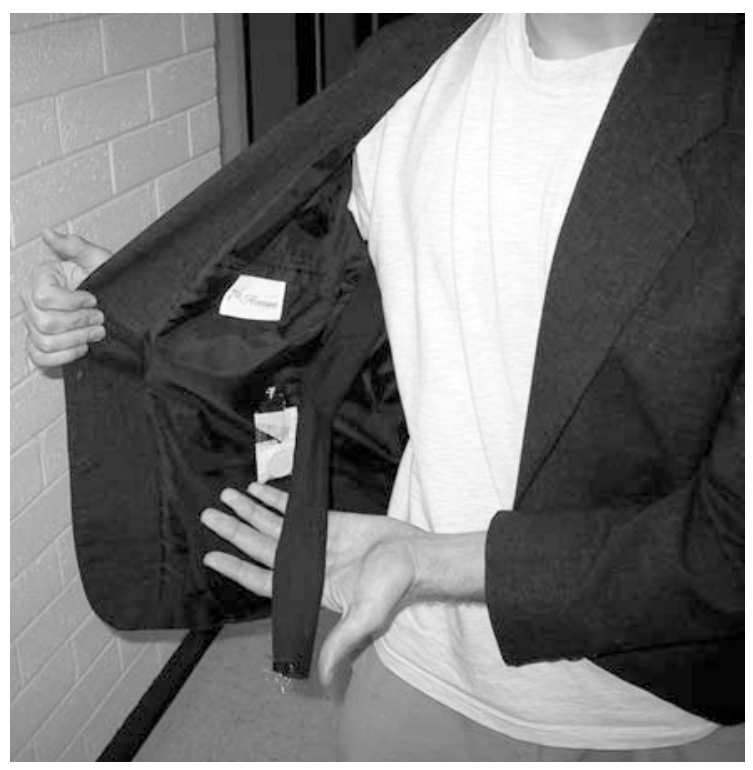

Fig. 9 In the jacket

and onHand watch PC used their own batteries. This configuration provided sufficient power to run the system for several hours of testing at a time. While sufficient for initial experimentation and user testing, the authors feel that due to the extremely high social weight and inconvenience of having to recharge units during the day any future versions of the e-Suit will require a minimum 19 hour runtime in order to be ready for rigorous user testing.

\subsection{The software}

The e-Suit software is driven by the functionality offered by current business tools. Integration of Microsoft 


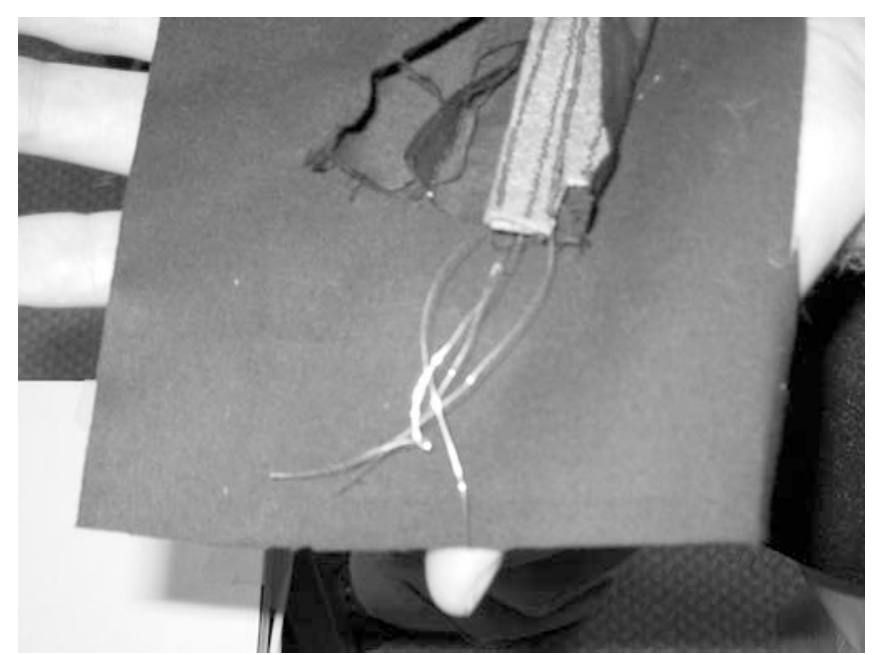

Fig. 10 A closeup

Outlook into the system takes advantage of a commercial grade PIM package capable of managing contact, task and appointment data across multiple, synchronised devices. This synchronisation allows users to consistently maintain the data in an environment that includes both a desktop PC that is used while the user is "in office", and handheld devices that are utilised when the user is in meetings or in other "out of office" scenarios.

A subset of this Microsoft Outlook functionality is considered for the e-Suit project. Remote/wireless synchronisation allows a mobile device mounted in the suit to be synchronised with appointments made on a desktop PC. Appointment manipulation functions (reminder acknowledgement and snooze) are mapped to capacitive buttons that allow operation with gestures of low social weight. Combined, these tasks allow a user to interact with Outlook in several scenarios.

Mobile devices participate in two separate roles within the e-Suit system. Firstly, it acts as a sensor that reacts to appointment data. Custom software interfaces with the Pocket Outlook Object Model (POOM) provided by the Outlook system, facilitating user interaction with the information. Secondly, the device acts as the system's master bus controller (MBC) with communication and co-ordination responsibilities. It is connected to the system bus via a standard RS-232 serial port, transmitting and receiving packets from a specifically designed protocol. Microsoft embedded Visual $\mathrm{C}++$ tools were used in the construction of this software.

\section{Discussion}

\subsection{What problems are caused by capacitive sensors?}

During the construction of the current e-SUIT keyboard, several methods of capacitive button detection were tried. All approaches, including the final set of buttons used with the e-SUIT, experienced the plethora of problems for which capacitive circuits are famous. In fact, the problems associated with capacitive detection circuits are exacerbated when used on the body; the body is an environment which has by its very nature a strong coupling to the circuit which is in a near constant state of flux. To obtain a reasonable performance, both hardware to provide hysteresis on the micro controllers input and software low pass filtering were required. Currently, further work is being done to add software auto calibration of the keyboard to help further reduce the effect of environmental noise.

\subsection{Where else can items be placed on the suit?}

Although the initial version of the e-SUIT was constrained to a jacket, a PDA and a watch this is by no means the limit to its domain. We predict that any successful clothing embedded wearable computer or interfaces must robustly and redundantly span multiple garments so as to not be defeated by a seasonal change in wardrobe or the simple removal of coat and hat upon entering a building. Additionally, the distribution of power and data within a successful multi-garment system must also be field-based to eliminate the need for cabling interconnecting the garments and limiting the user's range of motion.

\subsection{Where should the computing be on the e-SUIT?}

We have considered three options for the placement of the computing devices. Our current implementation has micro controllers embedded in the suit itself, a computer in the watch, a PDA in the suit pocket and a wireless LAN connection to a desktop computer. The current computer on a watch can communicate with Windows Outlook. One possibility is to remove the PDA and place the functionality of the PDA onto the watch computer. Assuming the wireless LAN connection can be made to the watch computer, this would be a viable option for the user. The user would have to make a choice between on the one hand not having to carry the PDA, and on the other hand having the reduced interaction capability of the watch computer. The watch computer has a much smaller screen and more limited input technology. This solution would also constrain the user to a particular watch computer. The embedded micro controller would allow the user to pick from a number of different watch and PDA vendors.

\subsection{How useful is sound?}

Due to the high $S W$ for sound displays that operate over public speakers, the use of sound must be used only in the correct social circumstances. Sound could be delivered to the user via an earpiece, but that would have a $S W$ of its own. We believe the way forward on using 
sound is a better contextual awareness of the system. Currently, the user controls when the sound is used as an alarm. If the system through the user's context could automatically determine if the $S W$ was appropriate for sound use, this would be a large bonus to the user. Sound makes a very good ambient interface, and does not require the user to change his visual focus.

\section{Future work}

\subsection{Context dependent social consequence}

Many of the components of social weight are based on the context of the social participants. A participant's social context guides his interpretation of the interaction, mediating social consequence. As a result differing social weights bear down between the user and each of the other social participants. Our current research has restricted its social context to the dyadic interactions illustrated earlier in Fig. 2, where a single social partner interacts with the user. Single partner interactions preclude any possible group social parallax in the partner's observations from biasing the result. To accurately characterise social weight future work will need to take place in a richer social context, containing multiple social partners as well as observers unknown to the user.

A workplace example of the impact of context on the formation of social consequence can be seen for users observed interrupting their social partners to interact with a device (e.g., PIMs, PDAs, pagers, etc.). Social partners are in a position to possibly benefit from the interruption as their questions get answered quickly or schedules are altered with minimal delay. Any perceived benefit from the disruption mitigates its social impact. Since observers lack these socially mitigating circumstances we believe they will be more likely to form harsher evaluations of the observed behaviour. In one context, the user is seen as responsive to his social partners and in the other he is not, with the social parallax occurring as a result of the context of the observer.

\subsection{Aggregate social consequence}

Prior stigma represents a potential source of aggregate social consequence to future interaction. Repeated negative, stigma-forming exposure to the user will invariably impact future interaction. For example within the workplace when users of a device are frequently observed interrupting their social partners, their co-workers may form harsh social evaluations. For our initial social weight research, the dyadic interactions were considered absent of prior social contact between the user and his social partner. Participants in our initial research were considered either recent acquaintances or unfamiliar strangers. This social model is in the minority within the workplace. While these context restrictions initially allowed characterization of social weight the effect of a history of social interaction upon the formation of social weight remains an unknown. Future work is required to accumulative effect prior interaction on the formation of device social weight.

\section{Conclusions}

The business world continually generates social situations with high economic and personal consequences. To mitigate the negative consequences accompanying technology use within a business context, that technology must be invisible to all social participants. Social transparency for an item of technology means allowing its user discrete, mobile access to information in an animate fashion. As a result social weight, with its corresponding interpersonal consequence, is of particular importance to business interactions.

In order to achieve social transparency within the business world, technology must be designed to fit the uniform of the business suit, a uniform that has enjoyed a stable design for close to two centuries. This paper defined the concept of social weight as a design consideration and presented the e-SUIT, a social weight research platform incorporated covertly within a traditional business suit. For proof of concept research within a business context, the e-SUIT was built upon commercially available software: Microsoft's Pocket Outlook. Our proof of concept testing showed access to commercially available enterprise applications with a distinct, user selectable strata of social weight. Users of the e-SUIT are given control of the selection of input and output devices along an awareness continuum, from one extreme of peripheral awareness information to the other of focal awareness information. As a result the e-SUIT allows its user to strike a balance between a given technology's derived benefit and its social consequence.

Acknowledgements The authors would like to acknowledge the assistance of Vina Brailsford for the lending of sewing equipment and practical tailoring advice.

\section{References}

1. Baxter LK (1997) Capacitive sensors: design and applications. IEEE Press, New York

2. Card SK, Moran TP and Newell A (1983) The psychology of human-computer interaction. Lawerence Erlbaum, Hillsdale, NJ

3. Cutrell E, Czerwinski M and Horvitz E (2001) Notification, disruption and memory: effects of messaging interruptions on memory and performance. In: Proceedings of the HumanComputer Interaction Conference, Tokyo, Japan, July 2001

4. Dey AK, Abowd GD (2000) CybreMinder: a context-aware system for supporting reminders. In: Proceedings of the 2nd International Symposium on Handheld and Ubiquitous Computing (HUC2K), Bristol, UK, 25-27 September 2000

5. Chenoune F et al. (1993) A history of mens' fashion. Flammarion, Paris

6. Filho RSS (2002) Awareness and privacy in mobile wearable computers. IPADS: interpersonal awareness devices. Final report, ICS-Information and Computer Science, 
UCI- University of California, Irvine. www.ics.uci.edu/ $\sim$ rsilvafi/papers/VirtualColocationFinalPaper.pdf.

7. Gemperle F, Ota N and Siewiorek D (2001) Design of a wearable tactile display. In: Proceedings of the 5th International Symposium on Wearable Computers, Zurich, Switzerland, 7-9 October 2001

8. Herstad J, van Thanh D and Audestad J (1999)Human centered mobile communication using contextual information. In: Proceedings of the International Workshop on Collaboration and Mobile Computing (CMC'99), Fukushima, Japan, September 1999

9. Matsucom Inc. (1999) onHand user's manual, 1st ed. Matsucom, Denver, $\mathrm{CO}$

10. Murray J (2000) Wearable computers in battle: recent advances in the land warrior system. In: Proceedings of the Fourth International Symposium on Wearable Computers, Atlanta, GA, 18-21 October 2000

11. Narayanaswami C, Raghunath MT (2000) Application design for a smart watch with a high resolution display. In: Proceedings of the Fourth International Symposium on Wearable Computers, Atlanta, GA, 18-21 October 2000

12. O'Conaill B, Frohlich D (1995) Timespace in the workplace: dealing with interruptions. In: Proceedings of the Conference on Human Factors and Computing Systems, Denver, CO, 7-11 May 1995
13. Pascoe J (1998) Adding generic contextual capabilities to wearable computers. In: Proceedings of the Second International Conference on Wearable Computers, Pittsburgh, PA, 19-20 October 1998

14. Post ER, Orth M, Russo PR and Gershenfeld N (2000) E-broidery: design and fabrication of textile-based computing. IBM Sys J 39:840-860

15. Robinson M, Kovalainen M and Auramäki E (2000) Diary as dialogue in papermill process control. Commun ACM 43:65-70

16. Sheridan J, Lafond-Favieres V and Newsletter W (2000) Spectators at a geek show: an ethographic inquiry into wearable computing. In: Proceedings of the Fourth International Symposium on Wearable Computers, Atlanta, GA, 18-21 October 2000

17. Siewiorek DP, Smailagic A, Bass L, Siegel J and Martin R (1998) Adtranz: a mobile computing system for maintenance and collaboration. In: Proceedings of the Second International Conference on Wearable Computers, Pittsburgh, PA, 19-20 October 1998

18. Thomas B, Grimmer K, Zucco J and Milanese S (2002) Where does the mouse go? An investigation into the placement of a body-attached touchPad mouse for wearable computers. Pers Ubiqu Comput 6:113-124 